\title{
ON THE GROWTH OF HOLOMORPHIC CURVES
}

\author{
NAN Wu AND ZuXING XuAN
}

\begin{abstract}
In this paper, we deduce some results which are generalizations of Petrenko, Fuchs, Niino, Bergweiler, Eremenko and Marchenko's work. We will estimate the deviation $b(a, G)$ of a holomorphic curve $G(z)$ with respect to a small holomorphic curve $a(z)$ and give an estimation of $L(r, a, G)$.
\end{abstract}

\section{Introduction}

In 1935, Valiron proved

PALey's CONJECTURE. An entire function $g(z)$ of order $\lambda$ satisfies

$$
\liminf _{r \rightarrow \infty} \frac{\log M(r, g)}{T(r, g)} \leq B(\lambda):= \begin{cases}\frac{\pi \lambda}{\sin \pi \lambda}, & \lambda \leq \frac{1}{2}, \\ \pi \lambda, & \lambda>\frac{1}{2} .\end{cases}
$$

The first complete proof for entire functions was given by Govorov in 1969 . Petrenko [20] proved this conjecture for meromorphic functions of finite lower order.

D. F. Shea (presented by Fuchs [7]) used the Valiron deficiency to give an improvement of Petrenko's theorem.

SHEA'S THEOREM ([7]). Let $f(z)$ be a meromorphic function of finite lower order $\mu$ and let $\Delta=\Delta(a, f)$ be the Valiron deficiency of $f$ at a. Then for each $a \in \mathbf{C}$, we have

$$
\liminf _{r \rightarrow \infty} \frac{\max _{|z|=r}\left\{\log ^{+}|f(z)-a|^{-1}\right\}}{T(r, f)} \leq B(\mu, \Delta),
$$

2000 Mathematics Subject Classification. 30D10 (primary), 30D20, 30B10, 34M05 (secondary). Key words and phrases. Holomorphic curve, Deviation, Defect, Subharmonic function.

The first author is supported in part by grants of NSF of China (Nos. 11231009, 11326086, 11371363). Xuan Zuxing is the corresponding author and he is supported in part by Beijing Natural Science Foundation (No. 1132013) and The Project of Construction of Innovative Teams and Teacher Career Development for Universities and Colleges Under Beijing Municipality (CIT and TCD20130513).

Received April 17, 2013. 
where $B(\mu, \Delta)$ is a constant number depending only on $\mu$ and $\Delta$ and is defined as

$$
B(x, \Delta)= \begin{cases}\pi x \sqrt{\Delta(2-\Delta)}, & \text { if } x \geq \frac{1}{2} \text { or if } 0<x<\frac{1}{2} \\ \pi x\left(\Delta \cdot \cot \pi x+\tan \frac{\pi x}{2}\right), & \text { and } \sin \frac{\pi x}{2} \geq \sqrt{\frac{\Delta}{2}},\end{cases}
$$

Remark 1. Paley's conjecture is a special case of Shea's theorem since $B(\mu, \Delta) \leq B(\mu)$.

In 1973, Niino [18] extended Shea's theorem to $n$-valued algebroid functions of finite lower order.

THEOREM 1.1 ([18]). Let $f(z)$ be an n-valued transcendental algebroid function with its $j$-th determination $f_{j}(z)$ of finite lower order $\mu$ and let $\Delta(\infty)=\Delta$ be the Valiron deficiency of $f(z)$ at $\infty$. Then, we have

$$
\liminf _{r \rightarrow \infty} \frac{\max _{|z|=r} \max _{1 \leq j \leq n}\left\{\log ^{+}\left|f_{j}(z)\right|\right\}}{T(r, f)} \leq B(\mu, \Delta) .
$$

In 1976, Petrenko [24] proved the the extension of Shea's theorem for a p-dimensional integral curve.

THEOREM 1.2 ([24]). If a p-dimensional integral curve $\vec{G}(z)$ has finite lower order $\mu$, then

$$
\liminf _{r \rightarrow \infty} \frac{\max _{|z|=r}\left\{\log \frac{\|\vec{G}(z)\|\|\vec{a}\|}{|\vec{G}(z) \cdot \vec{a}|}\right\}}{T(r, \vec{G})} \leq B(\mu, \Delta),
$$

for any fixed p-vector $\vec{a}$, where $\Delta=\Delta(\vec{a}, \vec{G})$.

In 1993, Bergweiler and Bock [5] studied the following analogue of Paley's conjecture for meromorphic functions of infinite lower order.

THEOREM $1.3([5])$. For a meromorphic function $f(z)$ of infinite lower order, we have

$$
\liminf _{r \rightarrow \infty} \frac{\max _{|z|=r}\left\{\log ^{+}|f(z)|\right\}}{r T_{-}^{\prime}(r, f)} \leq \pi
$$


In 1997, Eremenko [6] introduced the following quantity

$$
b(a, f)=\lim _{r \rightarrow \infty} \frac{\max _{|z|=r}\left\{\log ^{+}|f(z)-a|^{-1}\right\}}{A(r, f)}
$$

and proved an analogue of the defect relation.

THEOREM $1.4([6])$. Let $f(z)$ be a meromorphic function such that the set $\{a: b(a, f)>0\}$ contains more than one point. Then

$$
\sum_{a \in \hat{\mathbf{C}}} b(a, f) \leq 2 \pi
$$

In 1998, Marchenko [12] proved the analogue of Shea's theorem for $b(a, f)$.

THEOREM 1.5 ([12]). Let $f(z)$ be a meromorphic function of lower order $\mu$, where $0<\mu \leq \infty$. Then, for each $a \in \hat{\mathbf{C}}$,

$$
b(a, f) \leq \frac{B(\mu, \Delta)}{\mu},
$$

where $\Delta=\Delta(a, f)$ is the Valiron deficiency of a for $f(z)$.

In 2000, Marchenko [15] proved the following

THEOREM $1.6([15])$. Let $f(z)$ be a meromorphic function of lower order $0<\mu<\infty$ and order $0<\lambda \leq \infty$. For $0<\gamma<\infty$, put

$$
E_{0}(\gamma)=\left\{r: \max _{\theta \in[0,2 \pi)} \log ^{+}\left|f\left(r e^{i \theta}\right)-a\right|^{-1}<B(\gamma, \Delta(a, f)) T(r, f)\right\} .
$$

Then,

$$
\begin{aligned}
& \overline{\log \text { dens }} E_{0}(\gamma):=\limsup _{R \rightarrow \infty} \frac{1}{\log R} \int_{E_{0}(\gamma) \cap[1, R]} \frac{d t}{t} \geq 1-\frac{\mu}{\gamma}, \\
& \underline{\log \operatorname{dens}} E_{0}(\gamma):=\liminf _{R \rightarrow \infty} \frac{1}{\log R} \int_{E_{0}(\gamma) \cap[1, R]} \frac{d t}{t} \geq 1-\frac{\lambda}{\gamma} .
\end{aligned}
$$

The purpose of this paper is to extend Theorems 1.5 and 1.6 to the case of holomorphic curves dealing with small holomorphic curves.

The paper is arranged as follows. In Section 2, we give some notations and our results. In Section 3, we collect together some lemmas and auxiliary results. In Section 4, we prove Theorem 2.1 and then, in Section 5, we prove Theorem 2.2. 


\section{Notations and results}

We denote complex projective $n$-space by $P_{n} \mathbf{C}$ and the dual complex projective $n$-space by $\left(P_{n} \mathbf{C}\right)^{*}$. Let $G: \mathbf{C} \rightarrow P_{n} \mathbf{C}$ be a holomorphic curve and $\vec{G}=$ $\left(g_{0}, g_{1}, \ldots, g_{n}\right): \mathbf{C} \rightarrow \mathbf{C}^{n+1} \backslash\{0\}$ be its reduced representation, i.e., $g_{0}(z), g_{1}(z), \ldots$, $g_{n}(z)$ have no common zeros.

We define the characteristic function $T(r, G)$ of $G$ by

$$
T(r, G)=\frac{1}{2 \pi} \int_{0}^{2 \pi} \log \left\|\vec{G}\left(r e^{i \theta}\right)\right\| d \theta-\log \|\vec{G}(0)\|,
$$

where $\|\vec{G}(z)\|=\left(\sum_{j=0}^{n}\left|g_{j}(z)\right|^{2}\right)^{1 / 2}$. The order and lower order of a holomorphic curve $G(z)$ are defined as

$$
\lambda(G):=\limsup _{r \rightarrow \infty} \frac{\log T(r, G)}{\log r} ; \quad \mu(G):=\liminf _{r \rightarrow \infty} \frac{\log T(r, G)}{\log r} .
$$

For a holomorphic curve $a: \mathbf{C} \rightarrow\left(P_{n} \mathbf{C}\right)^{*}$ and $\vec{a}=\left(a_{0}, a_{1}, \ldots, a_{n}\right): \mathbf{C} \rightarrow$ $\mathbf{C}^{n+1} \backslash\{0\}$, which is its reduced representation, $\vec{G}(z) \cdot \vec{a}(z)$ is said to be free if $\vec{G}(z) \cdot \vec{a}(z) \not \equiv 0$. Let $n(r, a, G)$ denote the number of zeros (counting multiplicities) of the entire function

$$
F(z):=\vec{G}(z) \cdot \vec{a}(z)=\sum_{j=0}^{n} g_{j}(z) a_{j}(z) \not \equiv 0
$$

in the disk $\{|z| \leq r\}$. We denote by $N(r, a, G)$ the counting function of zeros of the entire function $F(z)$, that is, $N(r, a, G)=N(r, 0, F)$.

Under the assumption that $\vec{G}(z) \cdot \vec{a}(z) \not \equiv 0$, we define the approximating function $m(r, a, G)$ of $G$ and $a$ by

Put

$$
m(r, a, G)=\frac{1}{2 \pi} \int_{0}^{2 \pi} \log \frac{\left\|\vec{G}\left(r e^{i \phi}\right)\right\|\left\|\vec{a}\left(r e^{i \phi}\right)\right\|}{\left|\vec{G}\left(r e^{i \phi}\right) \cdot \vec{a}\left(r e^{i \phi}\right)\right|} d \phi .
$$

$$
T(r, a, G)=m(r, a, G)+N(r, a, G) .
$$

Applying the Jensen formula to the entire function $F(z)$, we have

$$
N(r, 0, F)=\frac{1}{2 \pi} \int_{0}^{2 \pi} \log \left|F\left(r e^{i \theta}\right)\right| d \theta-\log |F(0)| .
$$

This gives

$$
\begin{aligned}
T(r, a, G) & =m(r, a, G)+N(r, a, G) \\
& =\frac{1}{2 \pi} \int_{0}^{2 \pi} \log \left(\left\|\vec{G}\left(r e^{i \phi}\right)\right\|\left\|\vec{a}\left(r e^{i \phi}\right)\right\|\right) d \phi-\log |\vec{G}(0) \cdot \vec{a}(0)| \\
& =T(r, G)+T(r, a)+\log \frac{\|\vec{G}(0)\|\|\vec{a}(0)\|}{|\vec{G}(0) \cdot \vec{a}(0)|} .
\end{aligned}
$$


The Nevanlinna deficiency $\delta(a)=\delta(a, G)$ of $a$ respect to $G$ is defined by

$$
\delta(a, G)=\liminf _{r \rightarrow \infty} \frac{m(r, a, G)}{T(r, G)}=1+\limsup _{r \rightarrow \infty} \frac{T(r, a)-N(r, a, G)}{T(r, G)} .
$$

If $\delta(a, G)>0$ then we say that $a$ is a Nevanlinna deficient curve. We notice that if $a$ satisfies

$$
T(r, a)=o(T(r, G)), \quad r \rightarrow \infty,
$$

i.e., $a(z)$ is a small holomorphic curve of $G(z)$, then (2.4) is reduced to

$$
\delta(a, G)=1-\limsup _{r \rightarrow \infty} \frac{N(r, a, G)}{T(r, G)} .
$$

The Valiron deficiency $\Delta(a)=\Delta(a, G)$ of $a$ respect to $G$ is defined by

$$
\Delta(a, G)=\limsup _{r \rightarrow \infty} \frac{m(r, a, G)}{T(r, G)}=1+\liminf _{r \rightarrow \infty} \frac{T(r, a)-N(r, a, G)}{T(r, G)} .
$$

If $\Delta(a, G)>0$ then we say that $a$ is a Valiron deficient curve. We notice that if $a(z)$ is a small holomorphic curve of $G(z)$, then (2.7) is reduced to

$$
\Delta(a, G)=1-\liminf _{r \rightarrow \infty} \frac{N(r, a, G)}{T(r, G)} .
$$

The Petrenko deviation $\beta(a)=\beta(a, G)$ of $a$ respect to $G$ is defined by

$$
\beta(a, G)=\liminf _{r \rightarrow \infty} \frac{L(r, a, G)}{T(r, G)},
$$

where

$$
L(r, a, G)=\max _{|z|=r} \log \frac{\|\vec{G}(z)\|\|\vec{a}(z)\|}{|\vec{G}(z) \cdot \vec{a}(z)|} .
$$

The Bergweiler deviation $b(a)=b(a, G)$ of $a$ respect to $G$ is defined by

$$
b(a, G)=\liminf _{r \rightarrow \infty} \frac{L(r, a, G)}{r T_{-}^{\prime}(r, G)},
$$

where $T_{-}^{\prime}(r, G)$ is the left derivative of $T(r, G)$ at $r$.

If $T(r, a)=o(T(r, G))$, then $0 \leq \delta(a, G) \leq \Delta(a, G) \leq 1, \delta(a, G) \leq \beta(a, G)$.

Petrenko [21, 22, 24] once studied the deviation of the entire curve $\vec{G}(z)$ at a vector $\vec{a}$, but he did not study the deviation of the entire curve $\vec{G}(z)$ to a small entire curve $\vec{a}(z)$. In this paper we consider that $a(z)$ is a small holomorphic curve with respect to $G(z)$, i.e., $a(z)$ satisfies $T(r, a)=o(T(r, G))$, and we estimate the Bergweiler deviation $b(a, G)$ of a holomorphic curve $G(z)$ from a small holomorphic curve $a(z)$. 
THEOREM 2.1. Let $G(z)$ be a holomorphic curve of lower order $0<\mu \leq \infty$ and let $a(z)$ be a small holomorphic curve of $G(z)$. Then, we have

$$
b(a, G) \leq \frac{B(\mu, \Delta(a, G))}{\mu} .
$$

From Theorem 2.1, if $\Delta(a, G)=0$, then $b(a, G)=0$, which is Corollary 2.1 .

Corollary 2.1. Let $G(z)$ be a holomorphic curve of lower order $0<\mu \leq \infty$ and let $a(z)$ be a small holomorphic curve of $G(z)$. Then, we have

$$
B(G)=\{a: b(a, G)>0\} \subset E_{V}(G)=\{a: \Delta(a, G)>0\} .
$$

From the proof of Theorem 2.1, we can obtain the following.

COROLlaRY 2.2. Let $G(z)$ be a holomorphic curve of lower order $0<\mu \leq \infty$ and let $a(z)$ be a small holomorphic curve of $G(z)$. Then, we have

$$
\beta(a, G) \leq B(\mu, \Delta(a, G)) .
$$

Using Marchenko's method [15], we establish Theorem 2.2.

THEOREM 2.2. Let $G(z)$ be a holomorphic entire curve of lower order $0<\mu<$ $\infty$ and order $0<\lambda \leq \infty$ and let $a(z)$ be a small holomorphic curve of $G(z)$. For $0<\gamma<\infty$, put

$$
E(\gamma)=\{r: L(r, a, G)<B(\gamma, \Delta(a, G)) T(r, G)+o(T(r, G))\} .
$$

Then we have

$$
\begin{aligned}
& \overline{\log d e n s} E(\gamma):=\limsup _{R \rightarrow \infty} \frac{1}{\log R} \int_{E(\gamma) \cap[1, R]} \frac{d t}{t} \geq 1-\frac{\mu}{\gamma}, \\
& \underline{\log \operatorname{dens}} E(\gamma):=\liminf _{R \rightarrow \infty} \frac{1}{\log R} \int_{E(\gamma) \cap[1, R]} \frac{d t}{t} \geq 1-\frac{\lambda}{\gamma} .
\end{aligned}
$$

The method in this paper was firstly used by Fuchs [7] and Marchenko [15, $11,13]$ to investigate the growth of meromorphic functions.

\section{Some lemmas and auxiliary results}

Fuchs [7] amended the Petrenko's formula in [20] and established Lemma 3.1. We should notice that Lemma 3.1 is the Possion-Jensen formula in annulus.

LeMma 3.1 ([7]). Let $f(z)$ be a meromorphic function in the complex plane with its poles $\left\{b_{k}\right\}$. Then, for any $\gamma>1$ and $2 S<r<R / 2$, we have 


$$
\begin{aligned}
\log \left|f\left(r e^{i \phi}\right)\right| \leq & \frac{\gamma^{2}}{2 \pi} \int_{S}^{R} \frac{r^{\gamma} t^{\gamma-1}}{\left(r^{\gamma}+t^{\gamma}\right)^{2}} d t \int_{\phi-\pi / \gamma}^{\phi+\pi / \gamma} \log \left|f\left(t e^{i \theta}\right)\right| d \theta \\
& +\sum_{S \leq\left|b_{k}\right| \leq R} \log \left|\frac{r^{\gamma}+\left|b_{k}\right|^{\gamma}}{r^{\gamma}-\left|b_{k}\right|^{\gamma} \mid}\right| \\
& +\gamma K\left\{\left(\frac{S}{r}\right)^{\gamma} T(2 S, f)+\left(\frac{r}{R}\right)^{\gamma} T(R, f)\right\}
\end{aligned}
$$

where $\phi \in[0,2 \pi), K$ is an absolute constant number.

We also need the definition of the second kind of Pólya peaks.

Definition 3.1. Let $T(r)$ be an increasing function in $r>r_{0},\left\{r_{m}\right\}$ is called a sequence of Pólya peaks of the second kind of order $\mu$ for $T(r)$, if $r_{m} \rightarrow \infty$, and there exist two sequences $\left\{K_{m}\right\}$ and $\left\{\epsilon_{m}\right\}$, with $K_{m} \rightarrow \infty, \epsilon_{m} \rightarrow 0$, such that

$$
T(r)>\left(1-\epsilon_{m}\right)\left(r / r_{m}\right)^{\mu} T\left(r_{m}\right) \quad\left(r_{m} / K_{m}<r<K_{m} r_{m}\right) .
$$

Petrenko [19] gave an equality to treat with the error term, and Marchenko [10] amended his equality and established Lemma 3.2.

Lemma $3.2([10,19])$. Let $T(r)$ be a real function with finite lower order $\mu$, $\left\{S_{m}\right\}$ and $\left\{R_{m}\right\}$ be two sequences of real numbers such that

$$
\lim _{m \rightarrow \infty} S_{m}=\lim _{m \rightarrow \infty} R_{m}=\lim _{m \rightarrow \infty} R_{m} / S_{m}=\infty,
$$

$\left\{4 S_{m}\right\},\left\{4 R_{m}\right\}$ be two Pólya peaks of second kind of order $\mu$ for $T(r)$. Then for any $\varepsilon>0$, there exits $m_{0}(\varepsilon)>0$, such that for each $m>m_{0}$,

$$
T\left(4 S_{m}\right) S_{m}^{-\mu}+T\left(4 R_{m}\right) R_{m}^{-\mu}<\varepsilon \int_{2 S_{m}}^{R_{m} / 12} T(t) t^{-\mu-1} d t .
$$

Bergweiler [5] introduced some sequences generalizing Pólya peaks for a function $T(r)$ of infinite lower order. We recall the corresponding definition.

For all sequences $M_{j} \rightarrow \infty, \varepsilon_{j} \rightarrow 0$, there exist sequences $\rho_{j} \rightarrow \infty, \mu_{j} \rightarrow \infty$ such that for all $r$ satisfying the inequality $\left|\log \left(r / \rho_{j}\right)\right| \leq M_{j} / \mu_{j}$, we have the estimate

$$
T(r) \leq\left(1+\varepsilon_{j}\right)\left(\frac{r}{\rho_{j}}\right)^{\mu_{j}} T\left(\rho_{j}\right) .
$$

We can choose the sequences $\mu_{j}, M_{j}$ such that (see [5])

$$
\mu_{j}=o\left(\log ^{3 / 2} T\left(\rho_{j}\right)\right), \quad M_{j}=o\left(\log T\left(\rho_{j}\right)\right), \quad j \rightarrow \infty .
$$


We set

$$
P_{j}=\rho_{j} e^{-M_{j} / \mu_{j}}, \quad Q_{j}=\rho_{j} e^{M_{j} / \mu_{j}} .
$$

Then (3.2) holds for all $r \in\left[P_{j}, Q_{j}\right]$. We shall assume that $M_{j}>1$.

We consider the sets

$$
\begin{aligned}
& A_{j}=\left\{r \in\left[\rho_{j}, Q_{j}\right]: T(r) \leq \frac{1}{\sqrt{\mu_{j}}}\left(\frac{r}{\rho_{j}}\right)^{\mu_{j}} T\left(\rho_{j}\right)\right\}, \\
& B_{j}=\left\{r \in\left[P_{j}, \rho_{j}\right]: T(r) \leq \frac{1}{\sqrt{\mu_{j}}}\left(\frac{r}{\rho_{j}}\right)^{\mu_{j}} T\left(\rho_{j}\right)\right\} .
\end{aligned}
$$

Let

$$
\begin{array}{r}
R_{j}=\left\{\begin{array}{ll}
\min A_{j}, & \text { if } A_{j} \neq \emptyset, \\
Q_{j}, & \text { if } A_{j}=\emptyset,
\end{array} t_{j}= \begin{cases}\max B_{j}, & \text { if } B_{j} \neq \emptyset \\
P_{j}, & \text { if } B_{j}=\emptyset\end{cases} \right. \\
S_{j}=e^{-1 / \mu_{j}} R_{j}, \quad T_{j}=e^{-2 / \mu_{j}} R_{j} .
\end{array}
$$

Then

$$
t_{j}<\rho_{j}<T_{j}<S_{j}<R_{j}
$$

It is proved in [5] that

$$
\frac{T\left(R_{j}\right)}{R_{j}^{\mu_{j}}}+\frac{T\left(t_{j}\right)}{t_{j}^{\mu_{j}}}=o\left(\mu_{j} \int_{t_{j}}^{T_{j}} \frac{T(r)}{r^{\mu_{j}+1}} d r\right) .
$$

Moreover, it follows from inequality (19) in [5] that

$$
T\left(\rho_{j}\right) \leq T^{3 / 2}\left(t_{j}\right), \quad j \rightarrow \infty .
$$

\section{Proof of Theorem 2.1}

The proof results from the idea of Fuchs [7] and Marchenko [11].

CASE I. $\mu(G)<\infty$.

By the definition of $L(r, a, G)$, there exists a point $\theta_{0} \in[0,2 \pi)$ such that

$$
L(r, a, G)=\log \frac{\left\|\vec{G}\left(r e^{i \theta_{0}}\right)\right\|\left\|\vec{a}\left(r e^{i \theta_{0}}\right)\right\|}{\left|\vec{G}\left(r e^{i \theta_{0}}\right) \cdot \vec{a}\left(r e^{i \theta_{0}}\right)\right|} .
$$

For $z=r e^{i \theta_{0}}$, there exist two integers $k_{z}, l_{z}$ such that $1 \leq k_{z} \leq p, 1 \leq l_{z} \leq p$, and

$$
\begin{aligned}
L(r, a, G) & =\log \frac{\left\|\vec{G}\left(r e^{i \theta_{0}}\right)\right\|\left\|\vec{a}\left(r e^{i \theta_{0}}\right)\right\|}{\left|\vec{G}\left(r e^{i \theta_{0}}\right) \cdot \vec{a}\left(r e^{i \theta_{0}}\right)\right|} \\
& \leq \log ^{+}(n+1)+\log ^{+} \frac{\left|g_{k_{z}}\left(r e^{i \theta_{0}}\right)\right| \cdot\left|a_{l_{z}}\left(r e^{i \theta_{0}}\right)\right|}{\left|\vec{G}\left(r e^{i \theta_{0}}\right) \cdot \vec{a}\left(r e^{i \theta_{0}}\right)\right|} .
\end{aligned}
$$


Applying (3.1) to the meromorphic function $\left(g_{k_{z}}(\xi) a_{l_{z}}(\xi)\right) /(\vec{G}(\xi) \cdot \vec{a}(\xi))$ and combining with (4.1), we have

$$
\begin{aligned}
L(r, a, G)= & \log \frac{\left\|\vec{G}\left(r e^{i \theta_{0}}\right)\right\|\left\|\vec{a}\left(r e^{i \theta_{0}}\right)\right\|}{\left|\vec{G}\left(r e^{i \theta_{0}}\right) \cdot \vec{a}\left(r e^{i \theta_{0}}\right)\right|} \\
\leq & \frac{\gamma^{2}}{2 \pi} \int_{S}^{R} \frac{r^{\gamma} t^{\gamma-1}}{\left(r^{\gamma}+t^{\gamma}\right)^{2}} d t \int_{\theta_{0}-\pi / \gamma}^{\theta_{0}+\pi / \gamma} \log ^{+}\left|\frac{g_{k_{z}}\left(t e^{i \theta}\right) a_{l_{z}}\left(t e^{i \theta}\right)}{\vec{G}\left(t e^{i \theta}\right) \cdot \vec{a}\left(t e^{i \theta}\right)}\right| d \theta \\
& +\sum_{S \leq\left|b_{k}\right| \leq R} \log \mid \frac{r^{\gamma}+\left|b_{k}\right|^{\gamma}}{r^{\gamma}-\left|b_{k}\right|^{\gamma} \mid} \\
& +\gamma K\left\{\left(\frac{S}{r}\right)^{\gamma} T\left(2 S, \frac{g_{k_{z}} a_{l_{z}}}{\vec{G} \cdot \vec{a}}\right)+\left(\frac{r}{R}\right)^{\gamma} T\left(R, \frac{g_{k_{z}} a_{l_{z}}}{\vec{G} \cdot \vec{a}}\right)\right\}+\log ^{+}(n+1)
\end{aligned}
$$

where $\left\{b_{k}\right\}$ are the roots of $\vec{G}(\xi) \cdot \vec{a}(\xi)$. Here and in the below $K$ is a positive constant, which may have different values at different places. It is obvious that

$$
\begin{aligned}
\int_{\theta_{0}-\pi / \gamma}^{\theta_{0}+\pi / \gamma} \log ^{+}\left|\frac{g_{k_{z}}\left(t e^{i \theta}\right) a_{l_{z}}\left(t e^{i \theta}\right)}{\vec{G}\left(t e^{i \theta}\right) \cdot \vec{a}\left(t e^{i \theta}\right)}\right| d \theta & \leq m(t, a, G), \\
T\left(t, \frac{g_{k_{z}} a_{l_{z}}}{\vec{G} \cdot \vec{a}}\right) \leq m\left(t, \frac{g_{k_{z}} a_{l_{z}}}{\vec{G} \cdot \vec{a}}\right)+N(t, a, G) & \leq m(t, a, G)+N(t, a, G) \\
& =T(t, a)+T(t, G)+O(1) .
\end{aligned}
$$

Combining the above two equalities with (4.2), we have

$$
\begin{aligned}
& L(r, a, G) \leq \gamma^{2} \int_{S}^{R} \frac{r^{\gamma} t^{\gamma-1}}{\left(r^{\gamma}+t^{\gamma}\right)^{2}} m(t, a, G) d t+\sum_{S \leq\left|b_{k}\right| \leq R} \log \left|\frac{r^{\gamma}+\left|b_{k}\right|^{\gamma}}{r^{\gamma}-\left|b_{k}\right|^{\gamma}}\right| \\
&+\gamma K\left\{\left(\frac{S}{r}\right)^{\gamma}(T(2 S, G)+T(2 S, a))\right. \\
&\left.+\left(\frac{r}{R}\right)^{\gamma}(T(R, G)+T(R, a))\right\}+\log ^{+}(n+1) .
\end{aligned}
$$

We take $\gamma>\max (1,2 \mu)$ and divide the two sides of (4.3) by $r^{\mu+1}$ and integrate from $2 S$ to $R / 2$, for $-\gamma<p<\gamma, r>0$, by

$$
\gamma^{2} \int_{0}^{\infty} \frac{t^{\gamma-p-1}}{\left(t^{\gamma}+r^{\gamma}\right)^{2}} d t= \begin{cases}\frac{\pi p}{r^{\gamma+p} \sin (\pi p / \gamma)}, & \text { if } p \neq 0 \\ \gamma r^{-\gamma}, & \text { if } p=0\end{cases}
$$

and 


$$
\int_{0}^{\infty} \log \mid \frac{t^{\gamma}+|b|^{\gamma}}{t^{\gamma}-|b|^{\gamma} \mid} t^{-p-1} d t= \begin{cases}\frac{\pi}{p}|b|^{-p} \tan \frac{\pi p}{2 \gamma}, & \text { if } p \neq 0 \\ \frac{\pi^{2}}{2 \gamma}, & \text { if } p=0\end{cases}
$$

we can see that

$$
\begin{aligned}
\int_{2 S}^{R / 2} \frac{L(r, a, G)}{r^{\mu+1}} d r< & \frac{\pi \mu}{\sin (\pi \mu / \gamma)} \int_{S}^{R} \frac{m(r, a, G)}{r^{\mu+1}} d r+\pi \mu \tan \frac{\pi \mu}{2 \gamma} \int_{S}^{R} \frac{N(r, a, G)}{r^{\mu+1}} d r \\
& +K\left(S^{-\mu}(T(2 S, G)+T(2 S, a))\right. \\
& \left.+R^{-\mu}(T(2 R, G)+T(2 R, a))\right) .
\end{aligned}
$$

By the first fundamental theorem, namely (2.3), we have

$$
\begin{aligned}
m(r, a, G) & =T(r, a)+T(r, G)-N(r, a, G)+O(1) \\
& =(1+o(1)) T(r, G)-N(r, a, G)+O(1) .
\end{aligned}
$$

On the other hand, by the definition of Valiron deficiency, we get

$$
\begin{aligned}
N(r, a, G) & >(1-\Delta(a, G)-\varepsilon) T(r, G)+T(r, a) \\
& =(1-\Delta(a, G)-\varepsilon+o(1)) T(r, G) .
\end{aligned}
$$

By the choice of $\gamma, \gamma>2 \mu$, we have

$$
\tan \frac{\pi \mu}{2 \gamma}-\left(\sin \frac{\pi \mu}{\gamma}\right)^{-1}=-\cot \frac{\pi \mu}{\gamma}<0 .
$$

It follows from (4.6), (4.7) and (4.8) that

$$
\begin{aligned}
\int_{2 S}^{R / 2} \frac{L(r, a, G)}{r^{\mu+1}} d r< & \pi \mu\left[(\Delta(a, G)+\varepsilon) \cot \frac{\pi \mu}{\gamma}+\tan \frac{\pi \mu}{2 \gamma}+o(1)\right] \int_{S}^{R} \frac{T(r, G)}{r^{\mu+1}} d r \\
& +K(1+o(1))\left(\frac{T(2 S, G)}{S^{\mu}}+\frac{T(2 R, G)}{R^{\mu}}\right) .
\end{aligned}
$$

In particular, we choose $S=S_{m}, R=R_{m}$, where $S_{m}$ and $R_{m}$ are described in Lemma 3.2. And we notice that the quantity

$$
\int_{S_{m}}^{2 S_{m}}\left[T(r, G) / r^{\mu+1}\right] d r+\int_{R_{m} / 2}^{R_{m}}\left[T(r, G) / r^{\mu+1}\right] d r
$$

can be absorbed into the error term

$$
T\left(2 S_{m}, G\right) / S_{m}^{\mu}+T\left(2 R_{m}, G\right) / R_{m}^{\mu} .
$$

In view of Lemma 3.2, we obtain 


$$
\begin{aligned}
\int_{2 S_{m}}^{R_{m} / 2} \frac{L(r, a, G)}{r^{\mu+1}} d r< & \pi \mu\left[(\Delta(a, G)+\varepsilon) \cot \frac{\pi \mu}{\gamma}+\tan \frac{\pi \mu}{2 \gamma}+o(1)\right] \int_{2 S_{m}}^{R_{m} / 2} \frac{T(r, G)}{r^{\mu+1}} d r \\
& +K(1+o(1)) \varepsilon \int_{2 S_{m}}^{R_{m} / 2} \frac{T(r, G)}{r^{\mu+1}} d r \\
= & \left\{\pi \mu\left[(\Delta(a, G)+\varepsilon) \cot \frac{\pi \mu}{\gamma}+\tan \frac{\pi \mu}{2 \gamma}+o(1)\right]+K(1+o(1)) \varepsilon\right\} \\
& \cdot \frac{1+\varepsilon}{\mu} \int_{2 S_{m}}^{R_{m} / 2} \frac{r T_{-}^{\prime}(r, G)}{r^{\mu+1}} d r .
\end{aligned}
$$

Therefore, we have

$$
b(a, G)<\pi\left(\Delta(a, G) \cot \frac{\pi \mu}{\gamma}+\tan \frac{\pi \mu}{2 \gamma}\right) .
$$

Taking the maximum of the function $f(\gamma)=\Delta(a, G) \cot \frac{\pi \mu}{\gamma}+\tan \frac{\pi \mu}{2 \gamma}$, we can
obtain the result.

CASE II. $\mu(G)=\infty$.

Set

$$
\begin{gathered}
m^{*}\left(r e^{i \theta}, a, G\right)=\sup _{\operatorname{meas}(E)=2 \theta} \frac{1}{2 \pi} \int_{E} \log \frac{\left\|\vec{G}\left(r e^{i \varphi}\right)\right\|\left\|\vec{a}\left(r e^{i \varphi}\right)\right\|}{\left|\vec{G}\left(r e^{i \varphi}\right) \cdot \vec{a}\left(r e^{i \varphi}\right)\right|} d \varphi, \\
T^{*}\left(r e^{i \theta}, a, G\right)=m^{*}\left(r e^{i \theta}, a, G\right)+N(r, a, G),
\end{gathered}
$$

where $r \in(0, \infty), \theta \in[0, \pi], E \subset[0, \pi]$ is a Lebesgue measurable set. For any $0<t \leq \infty$, consider the set [9]

$$
F_{t}=\left\{r e^{i \varphi}: \log \frac{\left\|\vec{G}\left(r e^{i \varphi}\right)\right\|\left\|\vec{a}\left(r e^{i \varphi}\right)\right\|}{\left|\vec{G}\left(r e^{i \varphi}\right) \cdot \vec{a}\left(r e^{i \varphi}\right)\right|}>t\right\}, \quad \tilde{u}\left(r e^{i \varphi}\right)=\sup \left\{t: r e^{i \varphi} \in F_{t}^{*}\right\},
$$

where $F_{t}^{*}$ is the symmetric rearrangement of the set $F_{t}$. The function $\tilde{u}\left(r e^{i \varphi}\right)$ is non-negative and non-increasing in the interval $[0, \pi]$, even in $\varphi$ and for each fixed $r$ equimeasurable with $u\left(r e^{i \varphi}\right)$. Moreover, it satisfies the relations:

$$
\tilde{u}(r, 0)=\max _{|z|=r} \log \frac{\|\vec{G}(z)\|\|\vec{a}(z)\|}{|\vec{G}(z) \cdot \vec{a}(z)|}, \quad m^{*}\left(r e^{i \theta}, a, G\right)=\frac{1}{\pi} \int_{0}^{\theta} \tilde{u}\left(r e^{i \varphi}\right) d \varphi .
$$

From a result of Goldberg [8], we know that $\log \|\vec{G}(z)\|$ and $\log \|\vec{a}(z)\|$ are subharmonic functions, thus $\log (\|\vec{G}(z)\| \cdot\|\vec{a}(z)\|)$ is a subharmonic function. Since $\log |\vec{G}(z) \cdot \vec{a}(z)|$ is a subharmonic function, from Theorem $A^{\prime}$ in [2] and (2.2), the function $T^{*}\left(r e^{i \theta}, a, G\right)$ is subharmonic in the domain $K=\left\{r e^{i \varphi}: 0<\right.$ $r<\infty, 0<\varphi<\pi\}$, continuous in $\bar{K}=K \cup(-\infty, 0) \cup(0, \infty)$ and convex in $\log r$ for each fixed $\varphi \in[0, \pi]$. Moreover, 


$$
\begin{gathered}
T^{*}(r, a, G)=N(r, a, G), \quad T^{*}(-r, a, G)=T(r, G)+o(T(r, G)), \\
\frac{\partial}{\partial \theta} T^{*}\left(r e^{i \theta}, a, G\right)=\frac{\tilde{u}\left(r e^{i \theta}\right)}{\pi}, \quad 0<\theta<\pi .
\end{gathered}
$$

Suppose that $\alpha(r)$ is a real function. Set

$$
L \alpha(r):=\liminf _{h \rightarrow 0} \frac{\alpha\left(r e^{h}\right)+\alpha\left(r e^{-h}\right)-2 \alpha(r)}{h^{2}} .
$$

If $\alpha(r)$ is twice differentiable, then $L \alpha(r)=r \frac{d}{d r}\left(r \frac{d}{d r} \alpha(r)\right)$.

Since $T^{*}\left(r e^{i \varphi}, a, G\right)$ is a subharmonic function, we have

$$
L T^{*}\left(r e^{i \varphi}, a, G\right)+\frac{\partial^{2}}{\partial \varphi^{2}} T^{*}\left(r e^{i \varphi}, a, G\right) \geq 0 .
$$

Set

$$
\sigma(r)=\int_{0}^{\alpha} T^{*}\left(r e^{i \varphi}, a, G\right) \cos \mu_{j}(\varphi+\psi) d \varphi,
$$

where $0<\alpha<\pi /\left(2 \mu_{j}\right), \quad 0 \leq \psi \leq \pi /\left(2 \mu_{j}\right)-\alpha, \mu_{j}$ is defined as in (3.3). As $T^{*}\left(r e^{i \varphi}, a, G\right)$ is a convex function of $\log r$, applying Fatou's lemma, we obtain

$$
\begin{aligned}
L \sigma(r) & =L \int_{0}^{\alpha} T^{*}\left(r e^{i \varphi}, a, G\right) \cos \mu_{j}(\varphi+\psi) d \varphi \\
& \geq \int_{0}^{\alpha} L T^{*}\left(r e^{i \varphi}, a, G\right) \cos \mu_{j}(\varphi+\psi) d \varphi \geq 0 .
\end{aligned}
$$

It follows that $\sigma(r)$ is a convex function of $\log r$. Then, $r \sigma_{-}^{\prime}(r)$ is increasing on $(0, \infty)$. Thus, for almost all $r \in(0, \infty)$, we have

$$
L \sigma(r)=r \frac{d}{d r} r \sigma_{-}^{\prime}(r)
$$

Operating $\sigma(r)$ by $L$, and by (4.10), for almost all $r \in(0, \infty)$, we have

$$
\begin{aligned}
L \sigma(r)= & r \frac{d}{d r}\left(r \sigma_{-}^{\prime}(r)\right)=\int_{0}^{\alpha} L T^{*}\left(r e^{i \varphi}, a, G\right) \cos \mu_{j}(\varphi+\psi) d \varphi \\
\geq & -\int_{0}^{\alpha} \frac{\partial^{2}}{\partial \varphi^{2}} T^{*}\left(r e^{i \varphi}, a, G\right) \cos \mu_{j}(\varphi+\psi) d \varphi \\
= & -\left.\frac{\partial}{\partial \varphi} T^{*}\left(r e^{i \varphi}, a, G\right) \cos \mu_{j}(\varphi+\psi)\right|_{0} ^{\alpha} \\
& -\left.\mu_{j} T^{*}\left(r e^{i \varphi}, a, G\right) \sin \mu_{j}(\varphi+\psi)\right|_{0} ^{\alpha}+\mu_{j}^{2} \sigma(r) .
\end{aligned}
$$

We divide this by $r^{\mu_{j}+1}$ and integrate from $t_{j}$ to $T_{j}$ (where $t_{j}$ and $T_{j}$ are described by (3.4) and (3.5)): 


$$
\begin{aligned}
\int_{t_{j}}^{T_{j}}\left[\frac{\partial}{\partial \varphi}\right. & \left.T^{*}\left(r e^{i \varphi}, a, G\right)\right|_{\varphi=0} \cos \mu_{j} \psi-\left.\frac{\partial}{\partial \varphi} T^{*}\left(r e^{i \varphi}, a, G\right)\right|_{\varphi=\alpha} \cos \mu_{j}(\alpha+\psi) \\
& \left.-\mu_{j} T^{*}\left(r e^{i \varphi}, a, G\right) \sin \mu_{j}(\alpha+\psi)+\mu_{j} T^{*}(r, a, G) \sin \mu_{j} \psi\right] \frac{d r}{r^{\mu_{j}+1}} \\
\leq & \left(\frac{\sigma_{-}^{\prime}\left(T_{j}\right)}{T_{j}^{\mu_{j}-1}}+\mu_{j} \frac{\sigma\left(T_{j}\right)}{T_{j}^{\mu_{j}}}\right)-\left(\frac{\sigma_{-}^{\prime}\left(t_{j}\right)}{t_{j}^{\mu_{j}-1}}+\mu_{j} \frac{\sigma\left(t_{j}\right)}{t_{j}^{\mu_{j}}}\right) .
\end{aligned}
$$

By the definition of $\sigma(r)$, we obtain

$$
\sigma(r) \leq \frac{1}{\mu_{j}}(1+o(1)) T(r, G) .
$$

Since $r \sigma_{-}^{\prime}(r)$ is non-decreasing in $\left[t_{j}, T_{j}\right]$,

$$
\sigma\left(S_{j}\right)-\sigma\left(T_{j}\right) \geq \int_{T_{j}}^{S_{j}} \sigma_{-}^{\prime}(r) d r \geq T_{j} \sigma_{-}^{\prime}\left(T_{j}\right) \log \frac{S_{j}^{\prime}}{T_{j}}=\frac{1}{\mu_{j}} T_{j} \sigma_{-}^{\prime}\left(T_{j}\right),
$$

where $S_{j}$ is described as in (3.5). Thus,

$$
T_{j} \sigma_{-}^{\prime}\left(T_{j}\right) \leq \mu_{j} \sigma\left(S_{j}\right) \leq(1+o(1)) T\left(S_{j}, G\right) .
$$

On the other hand, by $r \sigma_{-}^{\prime}(r) \geq \sigma_{-}^{\prime}(1), r \geq 1$, we substitute the above inequality to (4.12), and in view of (3.6),

$$
\begin{aligned}
& \int_{t_{j}}^{T_{j}}\left[\left.\frac{\partial}{\partial \varphi} T^{*}\left(r e^{i \varphi}, a, G\right)\right|_{\varphi=0} \cos \mu_{j} \psi-\left.\frac{\partial}{\partial \varphi} T^{*}\left(r e^{i \varphi}, a, G\right)\right|_{\varphi=\alpha} \cos \mu_{j}(\alpha+\psi)\right. \\
& \left.\quad-\mu_{j} T^{*}\left(r e^{i \varphi}, a, G\right) \sin \mu_{j}(\alpha+\psi)+\mu_{j} T^{*}(r, a, G) \sin \mu_{j} \psi\right] \frac{d r}{r^{\mu_{j}+1}} \\
& \quad<\varepsilon \mu_{j} \int_{t_{j}}^{T_{j}} \frac{T(r, G)}{r^{\mu_{j}+1}} d r, \quad j \rightarrow \infty .
\end{aligned}
$$

Set $\psi=\frac{\pi}{2 \mu_{j}}-\alpha$ in the above inequality,

$$
\begin{aligned}
\int_{t_{j}}^{T_{j}} & {\left[\frac{L(r, a, G) \sin \mu_{j} \alpha}{\pi}-\mu_{j} T^{*}\left(r e^{i \alpha}, a, G\right)+\mu_{j} N(r, a, G) \cos \mu_{j} \alpha\right] \frac{d r}{r^{\mu_{j}+1}} } \\
& <\varepsilon \mu_{j} \int_{t_{j}}^{T_{j}} \frac{T(r, G)}{r^{\mu_{j}+1}} d r, \quad j \rightarrow \infty .
\end{aligned}
$$

On the other hand, by the definition of Valiron deficiency, i.e., (4.8) and $T^{*}\left(r e^{i \alpha}, \vec{a}, \vec{G}\right)<(1+o(1)) T(r, G)$, we have

$$
\int_{t_{j}}^{T_{j}} \frac{L(r, a, G)}{r^{\mu_{j}+1}} d r<\frac{\pi \mu_{j}}{\sin \mu_{j} \alpha}\left(1+\varepsilon-(1-\Delta(a, G)) \cos \mu_{j} \alpha\right) \int_{t_{j}}^{T_{j}} \frac{T(r, G)}{r^{\mu_{j}+1}} d r .
$$


Integrate the right side of the above by parts:

$\int_{t_{j}}^{T_{j}} \frac{L(r, a, G)}{r^{\mu_{j}+1}} d r<\frac{\pi}{(1-\varepsilon) \sin \mu_{j} \alpha}\left(1+\varepsilon-(1-\Delta(a, G)-\varepsilon) \cos \mu_{j} \alpha\right) \int_{t_{j}}^{T_{j}} \frac{r T_{-}^{\prime}(r, G)}{r^{\mu_{j}+1}} d r$.

There exists a sequence $r_{j} \in\left[t_{j}, T_{j}\right]$ such that

$$
L\left(r_{j}, a, G\right)<\frac{\pi}{(1-\varepsilon) \sin \mu_{j} \alpha}\left[1+\varepsilon-(1-\Delta(a, G)-\varepsilon) \cos \mu_{j} \alpha\right] r_{j} T_{-}^{\prime}\left(r_{j}, G\right)
$$

Hence,

$$
b(a, G)<\frac{\pi}{(1-\varepsilon) \sin \mu_{j} \alpha}\left[1+\varepsilon-(1-\Delta(a, G)-\varepsilon) \cos \mu_{j} \alpha\right] .
$$

Take $\alpha=\alpha_{j}$ such that $\cos \mu_{j} \alpha=1-\Delta(a, G)-\varepsilon$ :

Letting $\varepsilon \rightarrow 0$, we have

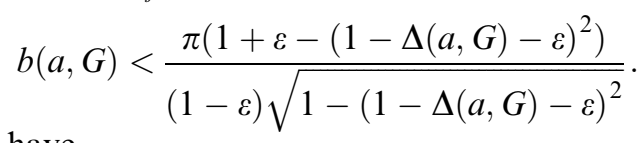

$$
b(a, G) \leq \pi \sqrt{\Delta(a, G)(2-\Delta(a, G))} .
$$

This completes the proof of Theorem 2.1.

\section{Proof of Theorem 2.2}

The proof results from the idea of Marchenko [15, 13]. We put the same significance on the same notations in the proof of Theorem 2.1. If $\gamma \leq \mu$, the theorem is obviously true. Suppose that $\gamma>\mu, \tau>0$ is a real number satisfying that $\mu<\tau<\gamma$. Choose $\alpha, \psi$ such that

$$
0<\alpha \leq \min \left(\pi, \frac{\pi}{2 \tau}\right), \quad-\frac{\pi}{2 \tau} \leq \psi \leq \frac{\pi}{2 \tau}-\alpha .
$$

Put

$$
\sigma(r)=\int_{0}^{\alpha} T^{*}\left(r e^{i \varphi}, a, G\right) \cos \tau(\varphi+\psi) d \varphi .
$$

For almost all $r \in(0, \infty),(4.10)$ implies that

$$
r \frac{d}{d r} r \sigma_{-}^{\prime}(r) \geq-\int_{0}^{\alpha} \frac{1}{\pi} \frac{\partial \tilde{u}\left(r e^{i \theta}\right)}{\partial \theta} \cos \tau(\theta+\psi) d \theta .
$$

Integrating twice by parts, we obtain

$$
\begin{aligned}
r \frac{d}{d r} r \sigma_{-}^{\prime}(r) \geq & -\frac{1}{\pi} \tilde{u}(r, \alpha) \cos \tau(\alpha+\psi)+\frac{1}{\pi} L(r, a, G) \cos \tau \psi \\
& -\tau T^{*}\left(r e^{i \alpha}, a, G\right) \sin \tau(\alpha+\psi)+\tau N(r, a, G) \sin \tau \psi+\tau^{2} \sigma(r) \\
:= & h_{\tau}(r)+\tau^{2} \sigma(r),
\end{aligned}
$$


Since $\tilde{u}\left(r e^{i \theta}\right)$ is decreasing in $\theta$, it follows that

$$
h_{\tau}(r)+\tau^{2} \sigma(r) \geq 0 .
$$

Dividing (5.1) by $r^{\tau+1}$ and integrating by parts over the interval $[r, R]$, we have

$$
\int_{r}^{R} \frac{h_{\tau}(t)}{t^{\tau+1}} d t \leq\left(\frac{R \sigma_{-}^{\prime}(R)}{R^{\tau}}+\tau \frac{\sigma(R)}{R^{\tau}}\right)-\left(\frac{r \sigma_{-}^{\prime}(r)}{r^{\tau}}+\tau \frac{\sigma(r)}{r^{\tau}}\right), \quad r_{0} \leq r \leq R,
$$

where $r_{0}$ is a constant number. Next we apply the method of Barry [3, 4]. Put

$$
\Phi(r):=-\int_{r}^{R} \frac{h_{\tau}(t)}{t^{\tau+1}} d t, \quad r_{0} \leq r \leq R .
$$

By (5.3), we deduce that

$$
\Phi(r) \geq-\frac{\sigma_{-}^{\prime}(R)}{R^{\tau-1}}-\tau \frac{\sigma(R)}{R^{\tau}}+\frac{\sigma_{-}^{\prime}(r)}{r^{\tau-1}}+\tau \frac{\sigma(r)}{r^{\tau}} .
$$

Put

$$
\Psi(r)=r^{\tau}\left[\Phi(r)+\frac{\sigma_{-}^{\prime}(R)}{R^{\tau-1}}+\tau \frac{\sigma(R)}{R^{\tau}}\right] .
$$

Combining (5.3) with (5.4), we derive that

$$
\Psi(r) \geq r \sigma_{-}^{\prime}(r)+\tau \sigma(r), \quad r_{0} \leq r \leq R .
$$

And combining (5.2) with (5.5), we derive that

$$
r \Psi^{\prime}(r)=\tau \Psi(r)+h_{\tau}(r) \geq \tau r \sigma_{-}^{\prime}(r)+\tau^{2} \sigma(r)+h_{\tau}(r) \geq \tau r \sigma_{-}^{\prime}(r), \quad r_{0}<r<R .
$$

We notice that for fixed $\theta, T^{*}\left(r e^{i \theta}, a, G\right)$ is an increasing function in $r$, so $\quad \sigma_{-}^{\prime}(r) \geq 0$. Set $A_{1}(\tau)=\left\{r \in\left[r_{0}, \infty\right): h_{\tau}(r)>0\right\}$. For $r \in A_{1}(\tau) \cap\left[r_{0}, R\right]$, $r \Psi^{\prime}(r)>\tau \Psi(r)>0$, that is

$$
\frac{\Psi^{\prime}(r)}{\Psi(r)}>\frac{\tau}{r}
$$

We deduce that

$$
\tau \int_{A_{1}(\tau) \cap\left[r_{0}, R\right]} \frac{d r}{r} \leq \int_{A_{1}(\tau) \cap\left[r_{0}, R\right]} \frac{\Psi^{\prime}(r)}{\Psi(r)} d r \leq \int_{r_{0}}^{R} \frac{\Psi^{\prime}(r)}{\Psi(r)} d r=\log \frac{\Psi(R)}{\Psi\left(r_{0}\right)} .
$$

The definition of $\Psi(r)$ yields

$$
\Psi(R)=R \sigma_{-}^{\prime}(R)+\tau \sigma(R) .
$$

And the definition of $\sigma(r)$ implies that

$$
\sigma(R) \leq \int_{0}^{\alpha}(1+o(1)) T(R, G) \cos \tau(\theta+\psi) d \theta \leq \pi(1+o(1)) T(R, G) .
$$


The monotonicity of $r \sigma_{-}^{\prime}(r)$ gives

$$
\sigma(2 R)-\sigma(R)=\int_{R}^{2 R} \sigma_{-}^{\prime}(r) d r=\int_{R}^{2 R} \frac{r \sigma_{-}^{\prime}(r)}{r} d r \geq R \sigma_{-}^{\prime}(R) \log 2 .
$$

This leads to

$$
R \sigma_{-}^{\prime}(R) \leq \frac{\sigma(2 R)}{\log 2} \leq \frac{\pi(1+o(1)) T(2 R, G)}{\log 2} .
$$

From (5.7) we can have a estimation:

$$
\Psi(R) \leq\left(\tau \pi+\frac{\pi}{\log 2}\right)(1+o(1)) T(2 R, G) .
$$

The inequality (5.6) gives

$$
\tau \int_{A_{1}(\tau) \cap\left[r_{0}, R\right]} \frac{d r}{r} \leq \log \left[\left(\tau \pi+\frac{\pi}{\log 2}\right)(1+o(1)) T(2 R, G)\right] .
$$

Thus $\overline{\log \operatorname{dens}} A_{1}(\tau) \leq \frac{\lambda}{\tau}, \underline{\log \operatorname{dens}} A_{1}(\tau) \leq \frac{\mu}{\tau} . \quad$ Put $E_{1}(\tau)=\left\{r \in\left[r_{0}, \infty\right): h_{\tau}(r) \leq\right.$ $0\}$, then $E_{1}(\tau)=\left[r_{0}, \infty\right) \backslash A_{1}(\tau)$, and

$$
\overline{\log \operatorname{dens}} E_{1}(\tau) \geq 1-\frac{\mu}{\tau}, \quad \underline{\log \operatorname{den} s} E_{1}(\tau) \geq 1-\frac{\lambda}{\tau} .
$$

Taking $\psi=\frac{\pi}{2 \tau}-\alpha$ in $h_{\tau}(r)$ gives

$$
h_{\tau}(r)=\frac{1}{\pi} L(r, a, G) \sin \tau \alpha-\tau T^{*}\left(r e^{i \alpha}, a, G\right)+\tau N(r, a, G) \cos \tau \alpha .
$$

From the definition of Valiron deficiency and the relation $T^{*}\left(r e^{i \alpha}, a, G\right) \leq$ $(1+o(1)) T(r, G)$, it follows that

$h_{\tau}(r) \geq \frac{1}{\pi}\{L(r, a, G) \sin \tau \alpha-\pi \tau[1-(1-\Delta(a, G)-\varepsilon) \cos \tau \alpha] T(r, G)\}-o(T(r, G))$.

For $r \in E_{1}(\tau), h_{\tau}(r) \leq 0$, we have

$$
L(r, a, G) \leq \frac{\pi \tau}{\sin \tau \alpha}[1-(1-\Delta(a, G)-\varepsilon) \cos \tau \alpha] T(r, G)+o(T(r, G)), \quad r \in E_{1}(\tau) .
$$

By the definition of $B(\tau, \Delta)$, we can prove that for $r \in E_{1}(\tau)$, the following holds:

$$
L(r, a, G) \leq B(\tau, \Delta(a, G)) T(r, G)+o(T(r, G))<B(\gamma, \Delta(a, G)) T(r, G)+o(T(r, G)) .
$$

Hence we deduce that $E_{1}(\tau) \subseteq E(\gamma)$. By the arbitrariness of $\tau$, we can obtain the desired result. 


\section{REFERENCES}

[1] A. BAERstein, Proof of Edrei's spead conjecture, Proc. London Math. Soc. 26 (1973), 418434.

[2] A. BAerstein, Integral means, univalent functions and circular symmetrization, Acta Math. 133 (1974), 139-169.

[3] P. Barry, On a theorem of Besicovitch, Quart. J. Math. Oxford. 14 (1963), 293-302.

[4] P. BARry, On a theorem of Kjeliberg, Quart. J. Math. Oxford. 15 (1964), 179-191.

[5] W. Bergweiler AND H. Bock, On the growth of meromorphic functions of infinite lower order, J. Anal. Math. 64 (1994), 327-336.

[6] A. ERemenko, An analogue of the defect relation for the uniform metric, Complex Var. 34 (1997), 83-97.

[ 7 ] W. H. J. Fuchs, Topics in Nevanlinna theory, Proc. of the NRL Conf. on Classical Function Theory, Naval Res. Lab., Washington, D.C., 1970, 1-32.

[8] A. A. Goldberg, Some questions of value distribution theory, Appendix to the Russian transl. of $\mathrm{H}$. Wittich, Neuere Untersuchungen über eindeutige analytische Funktionen, Fizmargiz, Moscow, 1960 (in Russian).

[9] W. K. Hayman, Multivalent functions, Cambridge University Press, Cambridge, 1958.

[10] I. I. Marchenko AND A. I. ShCherba, On the magnitudes of deviations of meromorphic functions, Math. Sb. 18-1 (1990), 3-24 (English transl. in Sb. Math. 69-1 (1991), 124).

[11] I. I. Marchenko, On the magnitudes of deviations and spreads of meromorphic functions of finite lower order, Math. Sb. 186-3 (1995), 85-102 (English transl. in Sb. Math. 186 (1995), 391-408).

[12] I. I. Marchenko, Growth of entire and meromorphic functions, Math. Sb. 189-6 (1998), 59-84 (English transl. in Sb. Math. 189 (1998), 875-899).

[13] I. I. Marchenko, On an analogue of the second main theorem for a uniform metric, Mat. Fiz. Anal. Geometr. 5 (1998), 212-227 (in Russian).

[14] I. I. MARchenko, On deviations and defects of meromorphic functions of finite lower order, Ukr. Math. J. 51 (1999), 889-898.

[15] I. I. Marchenko, On the Shea estimate for the magnitude of deviation of a meromorphic function, Izvestiya Vysshikh Uchebnykh Zavedenii. Matematika 6 (2000), 46-51 (in Russian).

[16] I. I. Marchenko, On the growth of entire and meromorphic functions, Math. Stud. 30 (2008), 139-146.

[17] I. G. Nikolenko AND I. I. Marchenko, On magnitudes of deviations of minimal surfaces, Vestnik Kharkov. Univ., Ser. Math. Mech. 444 (1999), 71-88.

[18] K. Nirno, On the growth of algebroid functions of finite lower order, Kodai Math. Sem. Rep. 25 (1973), 385-391.

[19] V. P. Petrenko, Growth of meromorphic functions, Izdat. Kharko. Univ., Kharkov, 1978 (in Russian).

[20] V. P. Petrenko, On the growth of meromorphic functions with finite lower order, Izv. Akad, Nauk SSSR. 33 (1969), 414-454 (in Russian).

[21] V. P. Petrenko and M. Hussain, On the growth of entire curves, Izv. Akad. Nauk SSSR, Ser. Mat. Tom 37 (1973), 467-478.

[22] V. P. Petrenko, Asymtptotic properties of meromorphic functions and entire curves, Ukr. Math. Zhur. 32 (1980), 773-780.

[23] V. P. Petrenko, Growth and distribution of values of algebroid functions, Matematicheskie Zametki 26 (1979), 513-522. 
[24] V. P. Petrenko, On the connection between the deviations and deficiencies in the sense of Valiron for entire curves and variable multivectors, Izv. Akad. Nauk SSSR, Ser. Mat. 40 (1976), 326-337.

$\mathrm{Nan} \mathrm{Wu}$

Department of Mathematics

SCHOOL OF SCIENCE

China University of Mining and Technology (Beijing)

BeIJING, 100083

People's Republic of ChINA

E-mail: wunan2007@163.com

Zuxing Xuan

Beijing Key Laboratory of Information Service Engineering

Department of General Education

BeiJing Union University

No.97 Bei Si Huan Dong RoAd

ChaOYANG District

BeIJING, 100101

People's Republic of ChinA

E-mail: xuanzuxing@ss.buaa.edu.cn 